\title{
Evaluando desempeños: alineamiento estratégico y productividad
}

\author{
Armando Cuesta Santos / cuesta@ind.cujae.edu.cu \\ Universidad Tecnológica de La Habana (CUJAE) \\ La Habana, Cuba
}

Recibido: 31 de agosto de 2011

Aceptado: 24 de abril de 2012

\section{RESUMEN:}

Se reporta como un problema mundial actual la ruptura o inexistencia de las conexiones a nivel de empresas entre la estrategia, la gestión de recursos humanos y los indicadores de gestión organizacional. En la práctica empresarial cubana se ha manifestado igualmente. Superar en el plano técnico organizativo esa ruptura, es el objetivo principal de esta investigación. En el artículo se argumenta un procedimiento metodológico partiendo de la definición de competencia laboral a considerar en los perfiles de cargo, que conduce a superar la actual ruptura u oposición (versus), en tanto no alineamiento o no integración, entre la evaluación del desempeño individual y la evaluación del desempeño estratégico organizacional.

Palabras clave: evaluación del desempeño, competencias, productividad

\section{ABSTRACT:}

Actually is reported as a world problem to enterprise label the rupture or inexistence of the connections between the strategy, human resource management and the organizational management indicators. In the Cuban enterprise practice this is manifest too. Surpass this rupture on the technical and organizer plan, is the principal objective of this research. In this paper is argument one methodological process that begins with the definition of labour competency to consider into post profiles, that conduced to surpass the actual rupture or opposition (versus), or not alignment or not integration, between the performance individual appraisal and the performance organizational strategic appraisal.

Keywords: performance appraisal, competencies, productivity 


\section{INTRODUCCIÓN}

Como proceso clave de Gestión de Recursos Humanos (GRH), la evaluación del desempeño laboral individual ha implicado por lo general un proceder metodológico, considerando un conjunto de indicadores, para reflejar de la forma más sistemática y objetiva posible el desempeño de los empleados en la organización (Werther y Davis, 2001; Chiavenato, 2002; Dolan, Valle, Jackson \& Schuller, 2003; Boxall, Purcell \& Wright, 2007). En esta contemporaneidad es más importante aún esta evaluación del desempeño individual, porque ante la necesidad de desarrollar la gestión estratégica de los recursos humanos, se impone una evaluación del desempeño de los trabajadores alineada, integrada, o en correspondencia, con el desempeño estratégico organizacional en aras de mayor productividad.

En estudio conjunto realizado por The Boston Consulting Group (BCG) y la World Federation of Personnel Management Associations (WFPMA), dando respuesta a cómo afrontar los desafíos en RRHH en todo el mundo hasta el 2015, se expresó: "Si contemplamos la estrategia, los indicadores de gestión y los RRHH como los tres vértices de un triángulo, veremos que en la mayoría de las empresas los vínculos entre RRHH y estrategia así como entre RRHH y los indicadores de gestión están rotos o no existen" (BCG \& WFPMA, 2008).

En ese estudio, destacó la importancia actual de la evaluación del desempeño, aludiendo a la necesidad de una estrategia sobre esa evaluación, respecto a la cual indicaron: "El enfoque de la empresa en la gestión del desempeño individual, los indicadores de capital humano y los sistemas de incentivos deberán apoyar los objetivos corporativos globales" (BCG \& WFPMA, 2008).

En las empresas del país, se ha venido manifestando esa ruptura o inexistencia referida por esa encuesta mundial realizada en 2008 por las instituciones BCG y WFPMA, que abarcó a 83 países, recogiendo las opiniones de más de 4700 directivos. En un estudio nacional realizado por el investigador Alfredo Morales en 2006, se evidenció que "en la empresa cubana, la carencia de la integración de los procesos internos de la gestión de recursos humanos y de esta, a su vez, con la estrategia empresarial, en correspondencia con las necesidades y realidades del país, frena la productividad del trabajo", que tuvo 
como antecedente un estudio del Ministerio de Trabajo y Seguridad Social en 2005, llevado a cabo en 2178 empresas del país, que representaban el 87\% del total (Morales, 2006).

En el aludido estudio de 2008 se hace referencia a la necesidad de considerar indicadores tangibles e intangibles en esa evaluación (destacando entre los intangibles el liderazgo y el compromiso de los empleados). No es netamente una evaluación económica o de productividad-no obstante su trascendencia-, sino también de indicadores intangibles vinculados al desarrollo humano. Interesa el desarrollo o crecimiento económico, pero cada vez más, interesa junto al crecimiento o desarrollo humano.

La importancia estratégica de la consideración anterior viene enfatizándola el Programa de las Naciones Unidas para el Desarrollo (PNUD), desde el "Informe sobre Desarrollo Humano", que a partir de 1990 viene emitiendo:

\begin{abstract}
"La verdadera riqueza de una nación está en su gente". Con estas palabras, el Informe sobre Desarrollo Humano de 1990 comenzó a abogar firmemente por un nuevo enfoque desde el cual afrontar el desarrollo. Hoy, su misión inicial de crear un entorno propicio para que las personas disfruten de una vida saludable, prolongada y creativa puede parecer más que evidente, pero no siempre ha sido así. En los últimos 20 años, esta publicación ha tenido como objetivo central enfatizar que el desarrollo se trata fundamentalmente de las personas (...) Por tal motivo y como alternativa explícita al PIB, se creó un índice sencillo-el Índice de Desarrollo Humano (IDH)-concentrado en la longevidad, la educación básica y el ingreso mínimo necesario. (PNUD, 2010)
\end{abstract}

En la segunda encuesta mundial que realizaron de conjunto la BCG y la WFPMA, en 2010, la evaluación del desempeño de la fuerza de trabajo pasó en su importancia común de un lugar 9, que ocupaba en la primera encuesta de 2008, a un lugar 4 de importancia. El giro de la marcha en 2010 de las empresas, en respuesta a la crisis económica, hizo mayor énfasis en aspectos evaluativos de productividad de la fuerza de trabajo que en aspectos asociados a las capacidades de los empleados, tales como la gestión del balance entre trabajo y calidad de vida, o en la gestión del cambio y la cultura (BCG \& WFPMA, 2010). 
Es un problema actual que la evaluación del desempeño individual sea opuesta (versus) a la evaluación estratégica, en tanto no están alineadas, integradas, o en correspondencia, oponiéndose o frenando la productividad del trabajo. Es problema común a empresas de distintos países del mundo. La ruptura entre estrategia, GRH y sus indicadores, es un problema que debe superarse. Y mediante las investigaciones implicadas en tesis doctorales que orientó este autor, que se refieren en el epígrafe siguiente y que se reflejará en estudio de caso, se ha apreciado su superación mediante el diseño de un procedimiento metodológico implicando que la evaluación del desempeño individual-conteniendo conductas estratégicas-, se manifieste alineada a la evaluación organizacional estratégica, posibilitando aumento de productividad. Argumentar esa superación, es objetivo principal de este artículo.

Que el desempeño individual, en particular la productividad del empleado, tribute considerando conductas estratégicas al alcance de los objetivos estratégicos de la organización, es algo que debe considerar estrictamente el sistema de evaluación del desempeño que se diseñe en estos tiempos. Comprendiendo a la vez un conjunto de indicadores tangibles e intangibles, implicando el crecimiento económico (productividad) y el desarrollo humano a la vez. Y ello requiere de un conjunto de elementos técnico organizativos, expresados en primer lugar mediante el análisis y diseño de cargos de trabajo, del cual se han de derivar los perfiles de cargo por competencias laborales, a su vez precedidos por el estudio de procesos de trabajo en función de la dirección estratégica asumida, considerando un control de gestión estratégica.

El objetivo fundamental de este trabajo es superar en el plano técnico organizativo la ruptura entre estrategia, GRH y sus indicadores, argumentando un procedimiento metodológico para que la evaluación del desempeño individual tribute al desempeño estratégico cuya evaluación refiera el alineamiento junto a mayor productividad. 


\section{Consideraciones teóRICO METOdológICAS fundamentales}

A la solución del problema relativo a la oposición actual entre la evaluación del desempeño individual y la evaluación estratégica organizacional-en tanto no hay alineamiento, integración, o correspondencia entre el desempeño individual y el desempeño estratégico organizacional-, manifestándose ruptura entre el tributo de la primera a la última, se viene contribuyendo desde hace algunos años, alcanzándose hoy una integración metodológica a partir fundamentalmente de la tutoría de investigadores que desarrollaron tesis doctorales en el ámbito del desempeño laboral y la gestión de recursos humanos y del conocimiento (Morales, 2006; De Miguel, 2007; Ramos, 2008; Soltura, 2009; Hernández, 2009; Valencia, 2010). En un conjunto de empresas cubanas y colombianas se verificaron concepciones y métodos, que se reflejan ahora integradas en el proceder metodológico que será presentado e ilustrado en lo esencial mediante estudio de caso, cuya argumentación radica en las consideraciones siguientes.

La concepción de base fue la de competencia laboral, desarrollada por el investigador Alfredo Morales, que llegó a ser refrendada en las normas cubanas NC 3000-3002: 2007 sobre gestión de capital humano-en cuya elaboración este autor participó como experto(NC 3000, 2007; NC 3001, 2007; NC 3002, 2007).

La definición de competencias laborales dada en esas normas cubanas, es la siguiente:

3.23 Competencias laborales. Conjunto sinérgico de conocimientos, habilidades, experiencias, sentimientos, actitudes, motivaciones, características personales y valores, basado en la idoneidad demostrada, asociado a un desempeño superior del trabajador y de la organización, en correspondencia con las exigencias técnicas, productivas y de servicios. Es requerimiento esencial que esas competencias sean observables, medibles y que contribuyan al logro de los objetivos de la organización. (NC 3000, 2007)

Para comprender de modo cabal ese concepto, y aprehender su relevancia metodológica, hay que asociarlo al desempeño laboral superior, por ello en esas mismas NC se delimitan los conceptos siguientes: 
3.33 Desempeño laboral adecuado. Rendimiento laboral y la actuación del trabajador, adecuado a los requerimientos establecidos para su cargo y expresa la idoneidad demostrada.

3.34 Desempeño laboral superior. Rendimiento laboral y la actuación superior del trabajador, con alto impacto económico y social, presente y futuro, identificado con las competencias laborales exigidas para su cargo. Este desempeño corresponde a las conductas estratégicas, es decir, a las competencias para lograr la estrategia de la entidad.

Esa definición de competencia laboral (competencia que corresponde al nivel de puesto o cargo) es holística, contraria a la atomización psicológica o a la fragmentación tayloriana del trabajador, superando el plano meramente funcional cognitivista, consecuente con el todo psicológico y con la unidad bio-psico-social en que se constituye la persona, implicando el desempeño superior. Y en la concepción de la misma se defiende la preeminencia de los valores humanos o éticos. Hay "desarrollo humano" en la misma, pretendiendo creatividad, dignidad, compromiso, entre otros valores.

Cuestión esencial en la determinación de las competencias a nivel de cargo o puesto de trabajo-en cuya materialización o desempeño por parte del trabajador está la génesis del valor agregado, y por tanto de la productividad-, atendiendo a esa conceptualización expresa en la NC, es el hecho de considerar "que contribuyan al logro de los objetivos de la organización”, así como al definir su inmanente complemento que es el desempeño laboral superior, precisar que ese desempeño corresponde a las "conductas estratégicas", es decir, a las competencias "para lograr la estrategia de la entidad". Muy cierto es lo que plantearon Robert S. Kaplan y David P. Norton, los autores del Cuadro de Mando Integral (CMI): "Para que la estrategia tenga verdaderamente significado para los empleados, las metas y objetivos personales deben estar alineados con los objetivos organizacionales" (Kaplan \& Norton, 2001).

Es imprescindible reconocer el carácter dinámico de este tipo de documento que es hoy el perfil de cargo por competencias. Al menos de este que se defiende aquí. Para el país se refrendó, que el "perfil de competencias del cargo" se adjuntará al "calificador de cargo" actual. En Cuba, el Calificador de cargo es un documento legal 
que tiene tres componentes: requisitos de conocimientos del cargo, funciones laborales y grupo escala salarial; y al mismo se adjuntará el "perfil de competencias". Cambian los objetivos estratégicos de la entidad laboral, e indefectiblemente tendrá que cambiar el perfil de competencias del cargo. Y tendrá que ser así puesto que el desempeño del cargo, que exige el desempeño individual de quien lo ocupe acorde al conjunto de competencias determinado, tendrá que poseer "conductas estratégicas", es decir, comportamientos que tributen al desempeño estratégico organizacional.

Y el proceso anterior se inicia con la planificación estratégica empresarial, una vez decidida la dirección estratégica: habiéndose definidos al menos la misión y los objetivos estratégicos. Con posterioridad se hacen los rediseños necesarios de los procesos de trabajo, para reajustar correspondencia de los mismos con la misión y objetivos estratégicos. $\mathrm{Y}$ atendiendo a los distintos procesos de trabajo reajustados o rediseñados se lleva a efectos el "Análisis y diseño de puestos”, con una salida que es el diseño ergonómico o físico del puesto o cargo, y otra salida que es el descriptor o calificador de cargo, al cual se le adjuntará el perfil de competencias determinado, sintonizado con los objetivos estratégicos a alcanzar. A la vez que se concibe esa planeación, se diseña el control de gestión estratégica, considerando indicadores intangibles especialmente asociados a la productividad (Valencia, 2010; Cuesta \& Valencia, 2010). Se diseña ese control de gestión estratégica que es el CMI desarrollado por Robert S. Kaplan y David P. Norton respecto al cual se ha experimentado en el país (Kaplan \& Norton, 2004; De Miguel, 2007; Hernández, 2009; Nogueira, Medina \& Nogueira, 2009).

No es con cualquier método que se determinan las competencias laborales de los distintos cargos. Tiene que ser mediante un método participativo y de expertos. Esos expertos tienen que haber vivenciado el cargo, y haber tenido éxito o un "desempeño laboral superior" en el mismo. Eso es esencial. Tendrán que conocer la misión y los objetivos estratégicos de la organización, traduciéndolos en "conductas estratégicas" a evidenciarse en el conjuto de competencias del cargo en cuestión; e iniciar la determinación de sus competencias una vez se haya determinado la misión del cargo alineado a la misión y objetivos organizacionales. La lógica que se ha propuesto por este autor 
al implementar las NC 3000-3002: 2007, va en orden descendente desde la consideración de las competencias de la organización, las competencias de procesos de trabajo, hasta las competencias laborales del cargo o puesto de trabajo (Cuesta, 2010a). Porque no es lineal el nexo desempeño individual con el organizacional, es zigzagueante, es complejo; y en el entramado que implica no linealidad, ha de manifestarse la necesaria sinergia entre las competencias del cargo, de procesos y de la organización.

Figura1: Método Delphi por rondas ponderado para la determinación de competencias laborales del cargo destacando pasos previos a su ejecución

Procedimiento de expertos (Delphi por rondas ponderado) para determinar las competencias laborales a nivel de cargos o puestos de trabajo

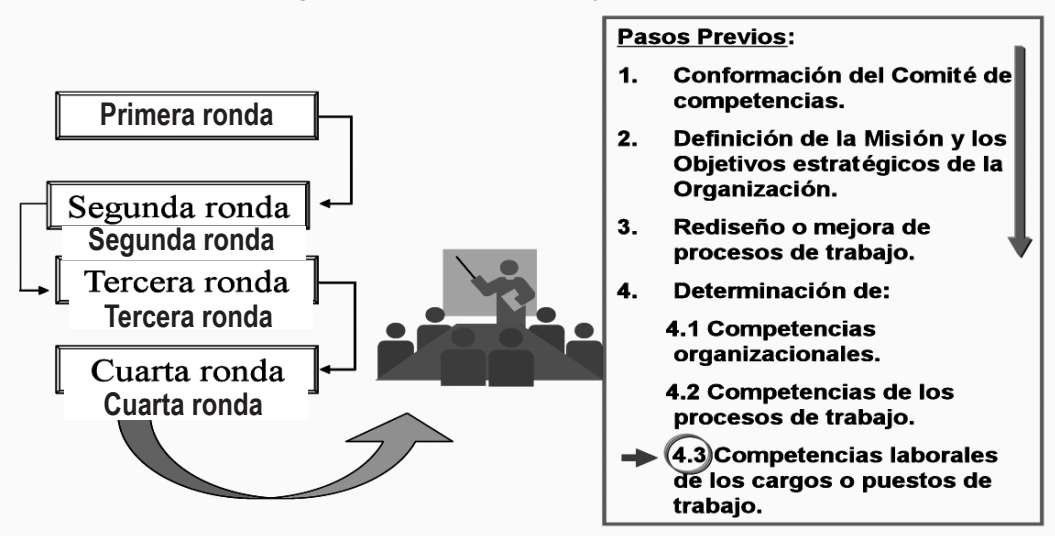

Esos expertos integrarán el "Comité de competencias", como lo requiere la NC 3001: 2007. Y metodológicamente eso es muy importante: todos habrán vivenciado un desempeño superior respecto al cargo al cual habrán de diseñar las competencias laborales. Hay muchísimas definiciones de competencia laboral, y muy respetables. Pero en la concepción que se defiende aquí, nunca puede perderse la perspectiva del nexo causal dado por "determinadas características de la persona... asociado a un desempeño exitoso", que fue la original precisada del trabajo conjunto de Richard Boyatzis con Da- 
vid McClelland (Boyatzis, 1982), con la cual mantiene plena consecuencia la que antes se adoptó, añadiéndole el tributo de "conductas estratégicas".

Sigue una reseña del proceder implicado en la aplicación del método que se propugna (Cuesta, 2001; Ramos, 2008; Cuesta, 2010b), el denominado "método Delphi por rondas ponderado" (reflejado en la Figura 1), con las peculiaridades que se exigen mediante la formulación del Cc. Su aplicación está referida al cargo, al punto 4.3 que se refleja en la Figura 1.

1. Desarrollo de la primera ronda, donde a cada experto (E) del grupo o Comité de competencias se le entrega una hoja de papel en la cual deben responder sin comentarios en el grupo. Pregunta: ¿Cuáles son las competencias que deben conformar el contenido del puesto $X$ ?

- Los especialistas que aplican el método listan todas las competencias, y después reducen el listado erradicando repeticiones o similitudes.

- Esos especialistas configuran la Tabla 1.

Tabla 1: Matriz de competencias (C) expresada por los expertos (E)

\begin{tabular}{|ccccc|}
\hline Competencia (C) & E1 & E2 & E3 & $\ldots$ E9 \\
\hline 1 & $X$ & - & $X$ & $X$ \\
2 & $X$ & $X$ & $X$ & - \\
3 & - & - & - & $X$ \\
$(\ldots)$ & & & & \\
17 & $X$ & $X$ & $X$ & \\
X: C relacionada por el experto. \\
$-:$ C no relacionada por el experto. \\
\end{tabular}

2. Segunda ronda. Se le entrega por separado a cada experto una hoja de papel donde es mostrada la matriz anterior (significada por la Tabla 1). Pregunta: ¿Está Ud. de acuerdo en que esas son verdaderamente las competencias para ese puesto? Con las que no esté de acuerdo márquelas con $N$.

- Una vez respondida la pregunta y recogidas las respuestas de todos los expertos, es determinado el nivel de concordancia a través de la expresión: 
$\mathbf{C c}=[1-(\mathrm{Vn} / \mathrm{Vt})] * 100$

donde,

Cc: coeficiente de concordancia expresado en porcentaje.

Vn: cantidad de expertos en contra del criterio predominante.

Vt: cantidad total de expertos.

- El procesamiento anterior implica la Tabla 2.

Tabla 2: Matriz de competencias depuradas con nivel de concordancia *

\begin{tabular}{|c|c|c|c|c|c|c|}
\hline Competencia & E1 & E2 & E3 & ... E9 & & Cc (\%) \\
\hline 1 & & & & & & 100 \\
\hline 2 & & & & & & 100 \\
\hline 3 & & $\mathrm{~N}$ & & & $\mathrm{~N}$ & 67 \\
\hline$(\ldots)$ & & & & & & \\
\hline 8 & $\mathrm{~N}$ & $\mathrm{~N}$ & & & & 78 \\
\hline
\end{tabular}

${ }^{*}$ Empíricamente, si resulta $\mathrm{Cc}^{3} 60 \%$ se considera aceptable la concordancia. Las $\mathrm{C}$ que obtuvieron valores $\mathrm{Cc}<60 \%$ se eliminaron por baja concordancia o poco consenso entre los $\mathrm{E}$. Puede apreciarse que de 17 competencias sólo quedaron 8.

3. Tercera ronda. Pregunta: ¿Qué ponderación o peso Ud. daría a cada una de las $C$, con el objetivo de ordenarlas atendiendo a su importancia en el desempeño laboral superior asociado a la misión del cargo? Aquí le es orientado a los $\mathrm{E}$ que el número 1 es la más importante, 2 la que sigue en importancia, hasta $n=8$, en este caso, que será la de menos importancia. Se insiste en que no deben ocurrir "ligas" o iguales ponderaciones a una misma competencia, pues se reduciría el poder de ordenamiento o discriminación.

- Recogidas las respuestas se ordenan las ponderaciones de acuerdo al valor de la sumatoria por filas indicada por $\mathrm{Rj}$. Esta variable después permitirá el ordenamiento por importancia, y con posterioridad se calcula el nivel de concordancia. 
Tabla 3: Ponderaciones de los expertos, orden de importancia (Rj) y nivel de concordancia $(\mathrm{Cc})$ relativo a $\mathrm{Rj}$

\begin{tabular}{|cccccccccccc|}
\hline Competencia & E1 & E2 & E3 & E4 & E5 & E6 & E7 & E8 & E9 & Rj & Cc (\%) \\
\hline 1 & 2 & 3 & 2 & 3 & 2 & 3 & 3 & 3 & 3 & 24 & 67 \\
2 & 1 & 2 & 1 & 2 & 1 & 2 & 1 & 1 & 1 & 12 & 67 \\
3 & 3 & 4 & 3 & 4 & 3 & 3 & 4 & 3 & 4 & 31 & 56 \\
4 & 4 & 5 & 4 & 5 & 4 & 5 & 5 & 5 & 6 & 43 & 56 \\
5 & 5 & 6 & 5 & 6 & 5 & 6 & 5 & 5 & 5 & 48 & 67 \\
6 & 6 & 7 & 6 & 7 & 6 & 7 & 6 & 7 & 7 & 59 & 56 \\
7 & 8 & 8 & 7 & 8 & 7 & 8 & 8 & 8 & 8 & 70 & 78 \\
8 & 7 & 1 & 8 & 1 & 8 & 1 & 1 & 8 & 7 & 42 & 45 \\
\hline
\end{tabular}

- De lo anterior resulta la Tabla 3. En la misma queda evidenciado cómo la competencia más importante es la 2, le continúa la 1 , y la menos importante es la 7 . $\mathrm{Y}$ ya es percibido el ordenamiento según los valores de $\mathrm{Rj}(\mathrm{C} 2, \mathrm{C} 1, \mathrm{C} 3, \ldots \mathrm{C} 7)$. Puede observarse, también en la Tabla 3, que en cuatro de las competencias no fue alcanzado $\mathrm{Cc}^{3} 60 \%$. Por tanto, hay que acudir a otra ronda.

4. Cuarta ronda. A los expertos se les hace llegar la Tabla 3, mostrándoles el ordenamiento alcanzado. Pregunta: ¿Está de acuerdo con las ponderaciones y el orden obtenido? Reflexione detenidamente. Puede modificar o mantener sus ponderaciones.

- Después se procede a realizar los cálculos de idéntico modo que en la tercera ronda.

Para no redundar numéricamente, sólo se expresará que de esta cuarta ronda se obtuvo una tabla similar a la 3, pero con todas las $\mathrm{C}$ ${ }^{3} 60 \%$, y manteniéndose el ordenamiento consignado en la Tabla 3. Entonces, es conclusión que hay un adecuado nivel de consenso y se 
arriba a las competencias para ese puesto, determinadas por ese grupo de expertos. Es logrado así, técnicamente argumentado, el perfil de competencias respecto al contenido de trabajo.

En esta línea del procesamiento estadístico es necesario señalar que ha sido recurrido el coeficiente de concordancia Kendall $W$ (Cuesta, 2001; Cuesta, 2010b). Por ello es denominada $R j$ a la sumatoria de ponderaciones por filas, variable con igual denominación en Kendall $W$. Tal estadígrafo supera al coeficiente Cc por su rigor estadístico matemático. Demuestra significación en el consenso con menos rondas. En una época resultaba engorrosa la aplicación de ese estadígrafo por la cantidad de cálculos implicados, pero hoy con $P C$ y programas como los de Microsoft disponible, es quehacer fácil y de pocos minutos, aparejando la bondad de pausas necesarias de reflexión o descanso para los expertos participantes.

En esta experiencia, enfrentar la tarea de definir las pautas de conducta o dimensiones de cada competencia, ha sido lo más complicado. Sin embargo, fluyó bien en el grupo su definición en esa experiencia. Al pedir que listaran entre dos o tres pautas de conducta o dimensiones del desempeño, respecto a cada competencia, la coincidencia fue impresionante. Hubo consenso en las reuniones para definir tales pautas que, en general, resultaron tres por cada competencia. Se apreciará en el epígrafe posterior, que esas pautas serán cinco, en orden creciente para indicar el "desempeño laboral superior" o de Excelente. Se ha trabajado llevando al Comité de competencias (Ramos, 2009) esos niveles o gradientes de las dimensiones.

\section{Diseño del PROCEdimiento Metodológico}

El diseño del procedimiento metodológico para alcanzar el alineamiento de la evaluación del desempeño individual con la evaluación del desempeño estratégico organizacional, se reseña en la Figura 2. 
Figura 2: Procedimiento metodológico para el alineamiento de la evaluación del desempeño individual con la evaluación del desempeño estratégico organizacional (resumen)

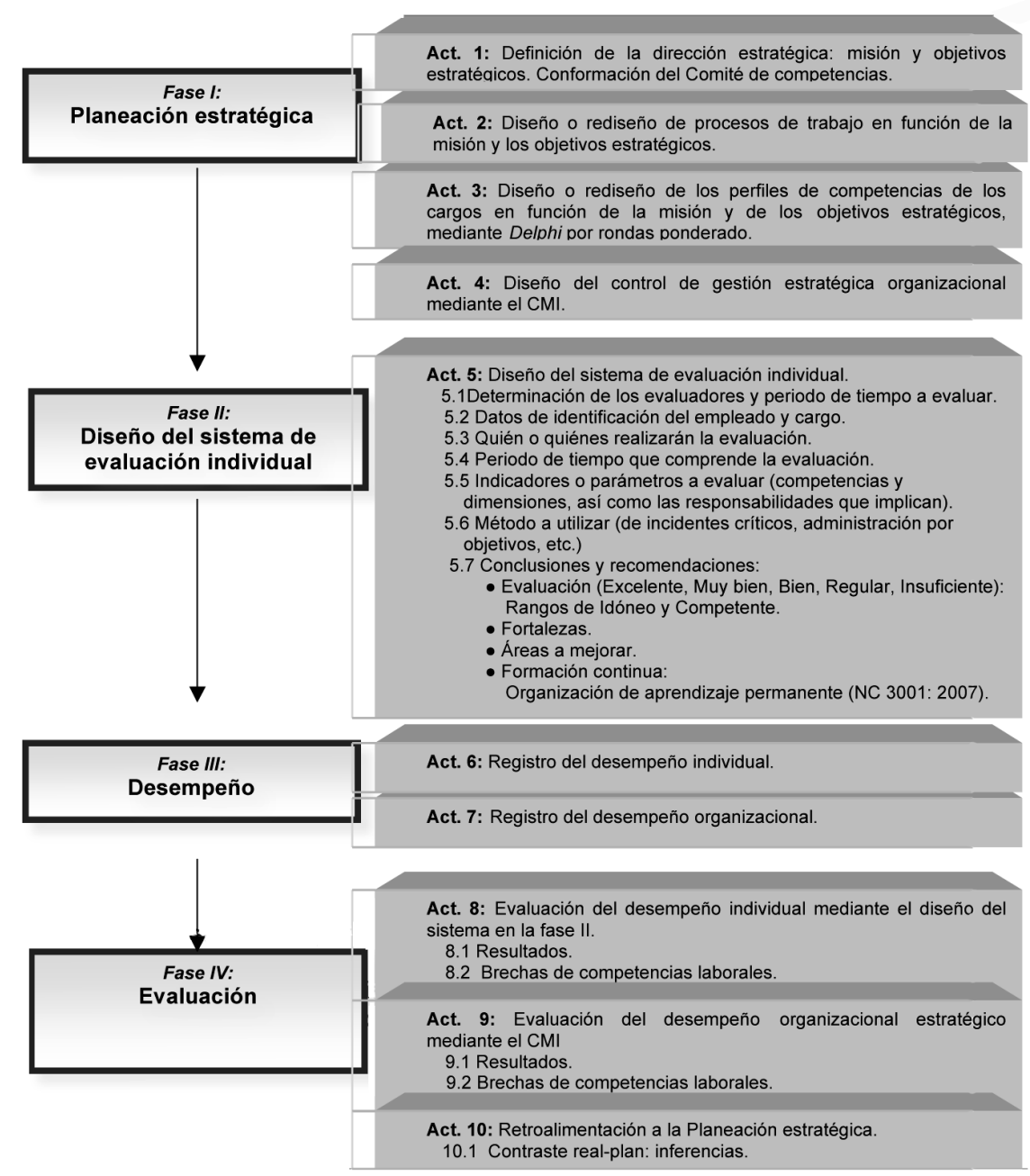

A continuación se ilustrará mediante estudio de caso, lo esencial del procedimiento metodológico, en la empresa denominada Cumpelet. Los objetivos estratégicos determinados para la empresa Cumpelet fueron los siguientes: 
1. Garantizar un $100 \%$ de asistencia a los servicios solicitados por los clientes.

2. Alcanzar la satisfacción de la clientela mediante el logro de la calidad requerida en los servicios.

3. Garantizar la optimización de los procesos clave de trabajo, referidos a la logística, la elaboración y la comercialización.

4. Captar los ingresos financieros correspondientes al 100\% de asistencia a los servicios solicitados.

5. Garantizar la formación continua mediante la asunción del concepto de organización que aprende.

6. Lograr que el $100 \%$ de los trabajadores alcance la idoneidad acorde a los perfiles de competencias de los cargos que ocupan, y así alcanzar la excelencia en el servicio.

Los valores de la empresa Cumpelet son: compromiso, honradez y cooperación. Las competencias organizacionales que conforman la empresa Cumpelet son:

- Alta preparación técnica de sus recursos humanos.

- Elevado compromiso con la institución.

- Honradez en su gente.

- Cooperación entre los integrantes.

La Tabla 4 expresa la relación de la misión y estrategia con los valores de la referida empresa y sus impactos (X) en los objetivos estratégicos de la empresa Cumpelet. 
Tabla 4: Relación de la misión con los valores y sus impactos (X) en los objetivos estratégicos de Cumpelet

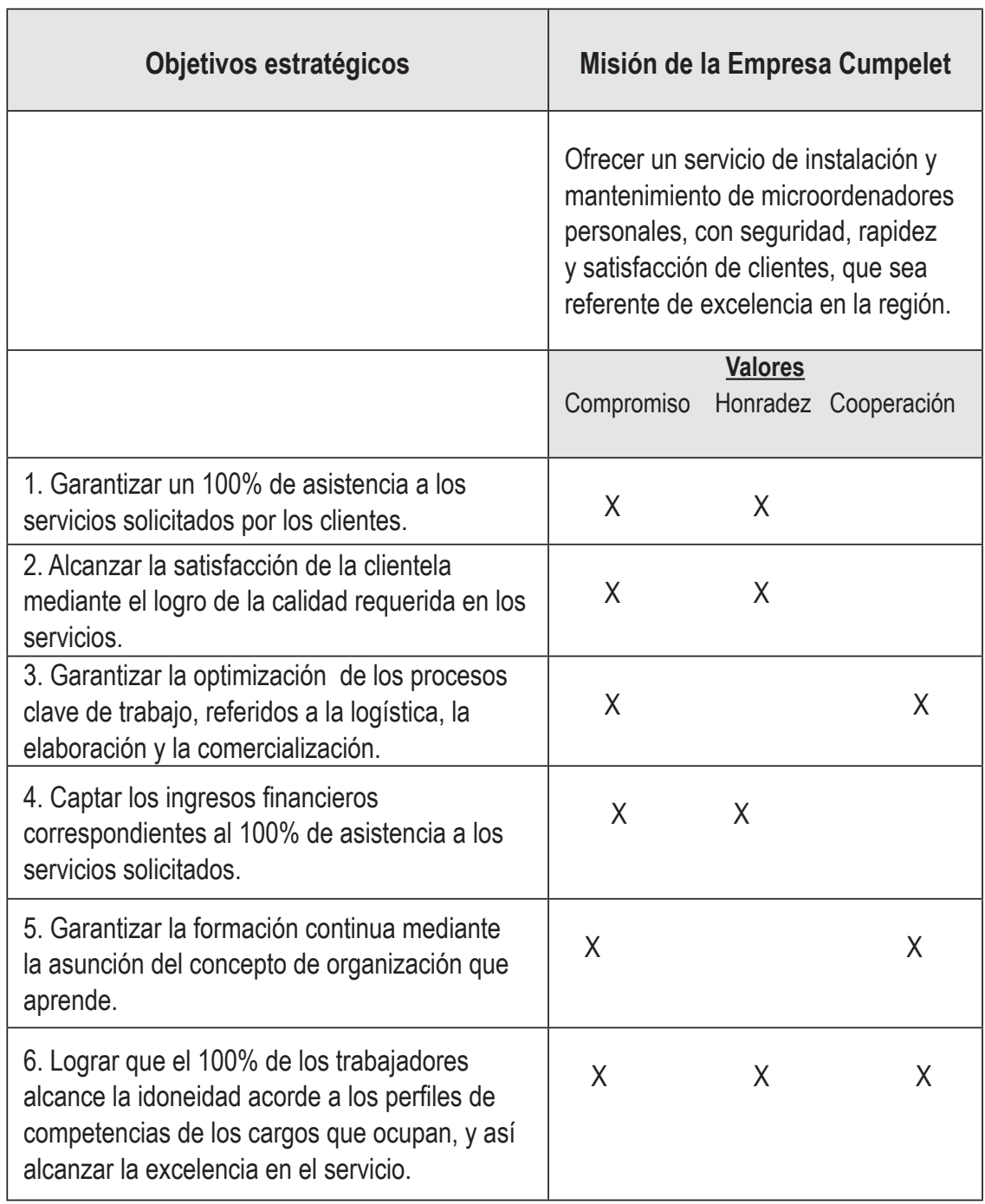


Figura 3: Mapa estratégico destacando enumerados los objetivos estratégicos en sus nexos causales

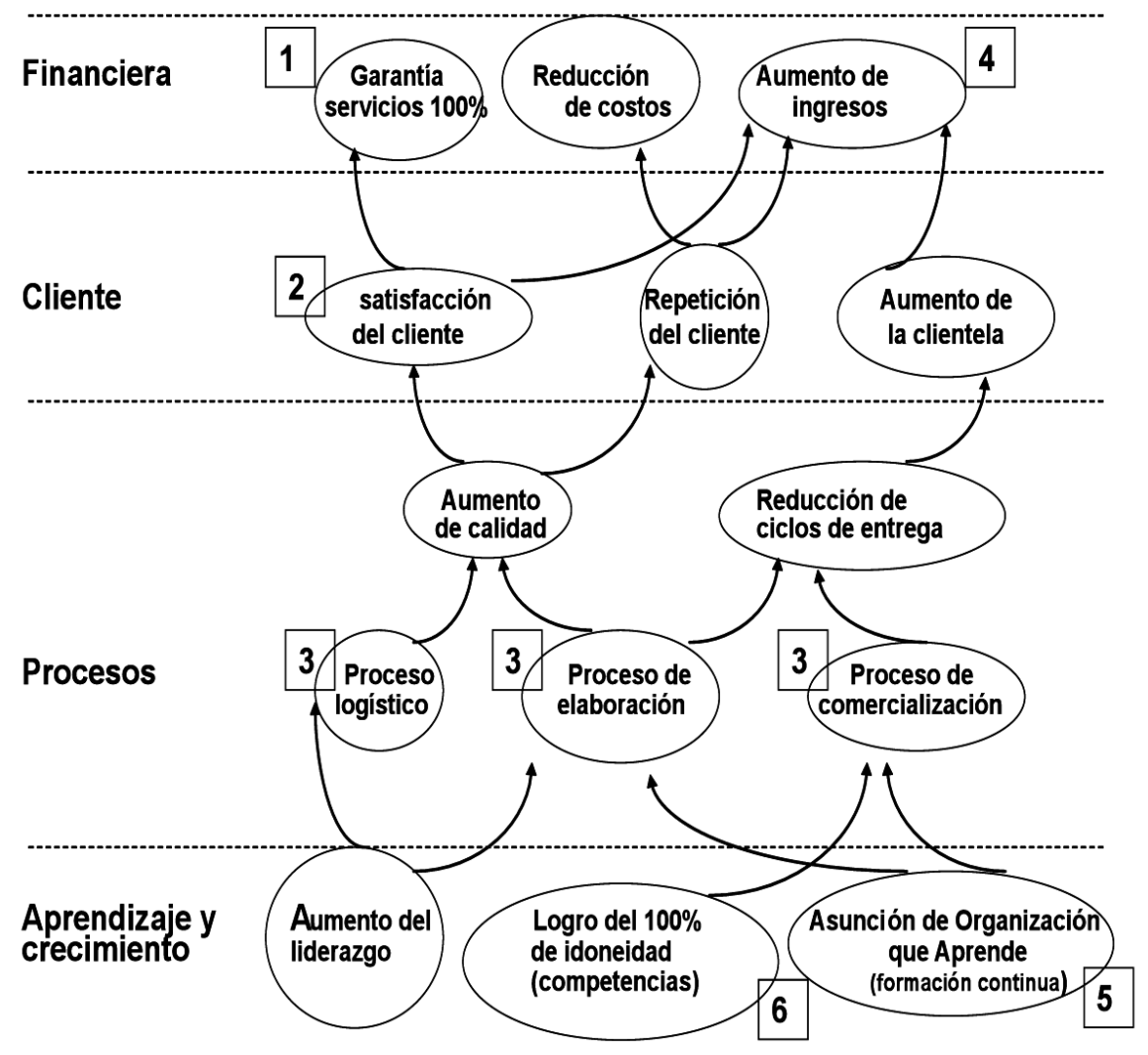

En la Figura 3 queda planteado el Cuadro de Mando Integral (CMI) de la empresa Cumpelet. La Tabla 5 consigna la derivación de los indicadores y metas de los objetivos estratégicos. 
EVALUANDO DESEMPEÑOS: ALINEAMIENTO ESTRATÉGICO Y PRODUCTIVIDAD

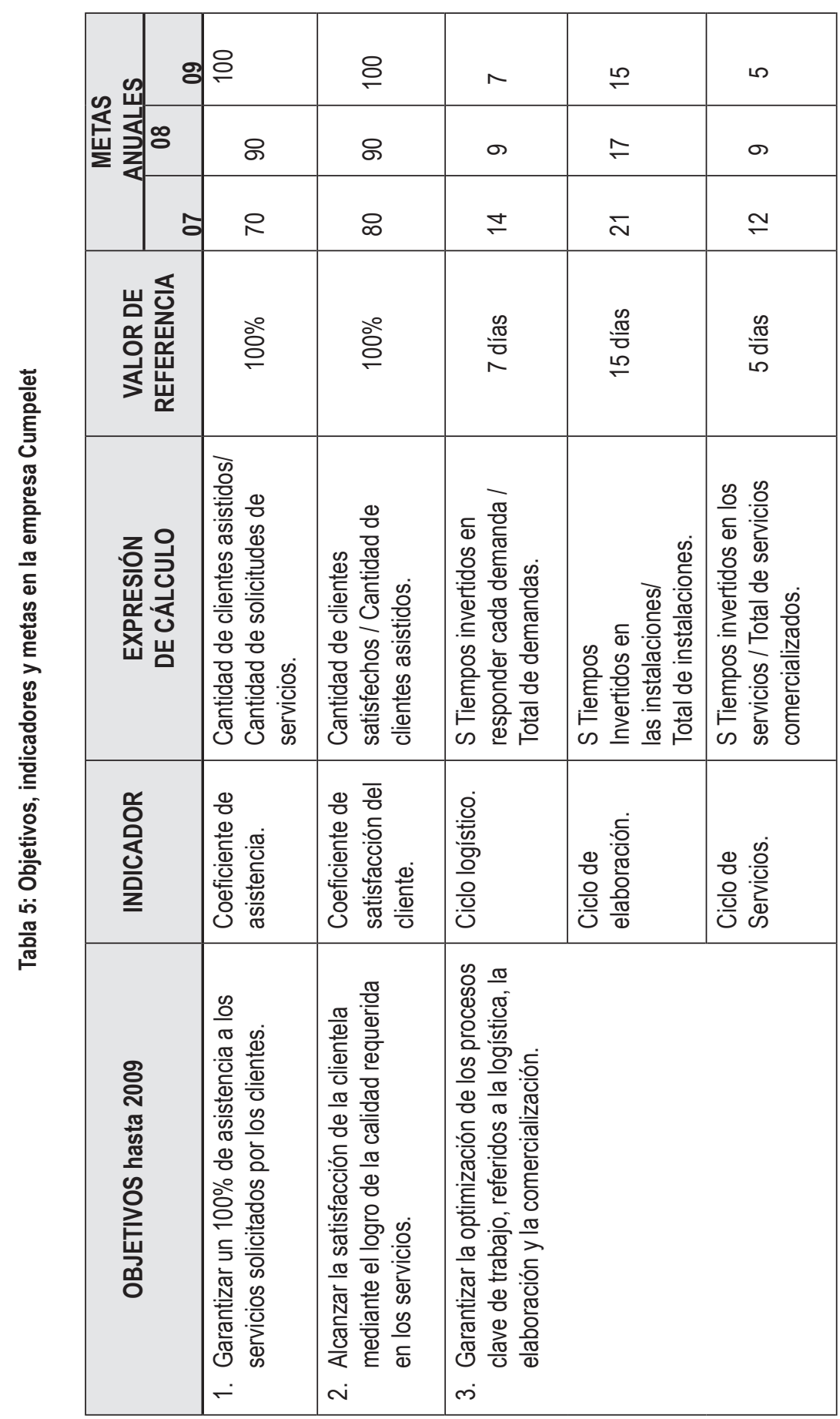


Cuesta Santos

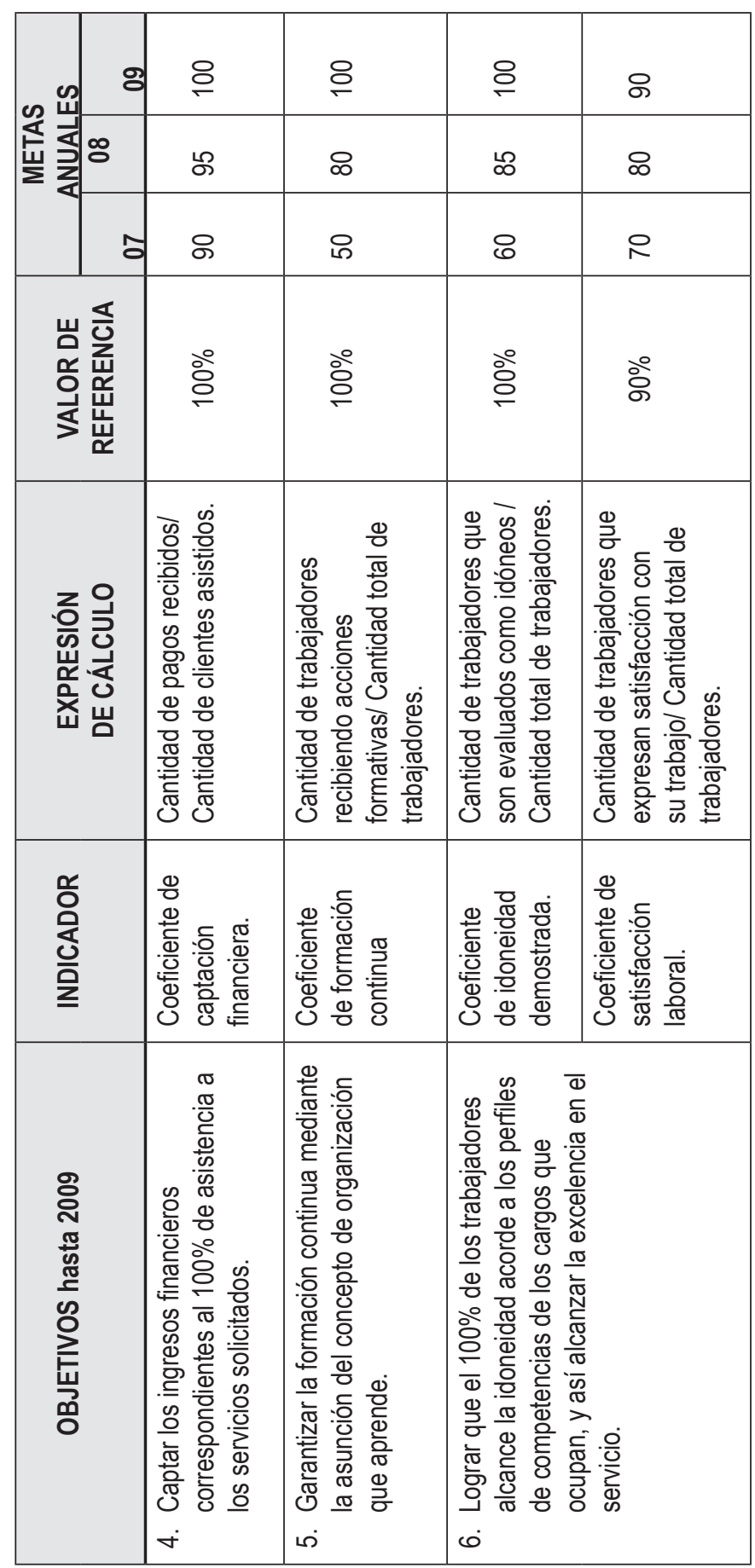

18 ISSN 1541-8561

FORUM EMPRESARIAL Vol. 17,1 (MAYO 2012) 
A continuación, reflejado en la Tabla 6, el sistema de evaluación del desempeño diseñado, para uno de los cargos de trabajo de la entidad laboral de servicio que se ha tomado como referencia. En ese cargo o puesto de trabajo se ubicaba la mayoría de los empleados de la empresa Cumpelet, devenidos empleados directos, dado su vínculo directo en la creación del plusvalor o el valor agregado.

Nótese cómo las siete (7) competencias laborales de ese cargo de trabajo, determinadas por el Comité de expertos o de competencias, responden a los seis (6) objetivos estratégicos trazados para Cumpelet, en tanto implican comportamientos o "conductas estratégicas" asociadas al logro de los mismos. Los niveles o grados (0-10) en que se manifiestan esas competencias, son las distintas pautas de conducta o dimensiones, significando indicadores de medición, operativización o aterrizaje de cada una de las competencias laborales. El diseño de esas dimensiones va in crescendo, para establecer los necesarios rangos del desempeño, desde Insatisfactorio o no idoneidad extrema (0-2), pasando a Idóneo (7-8, con "desempeño laboral adecuado") y hasta Competente (9-10, con "desempeño laboral superior"), ubicados estos dos nominadores del desempeño individual en las puntuaciones de Muy bueno y Excelente respectivamente. 


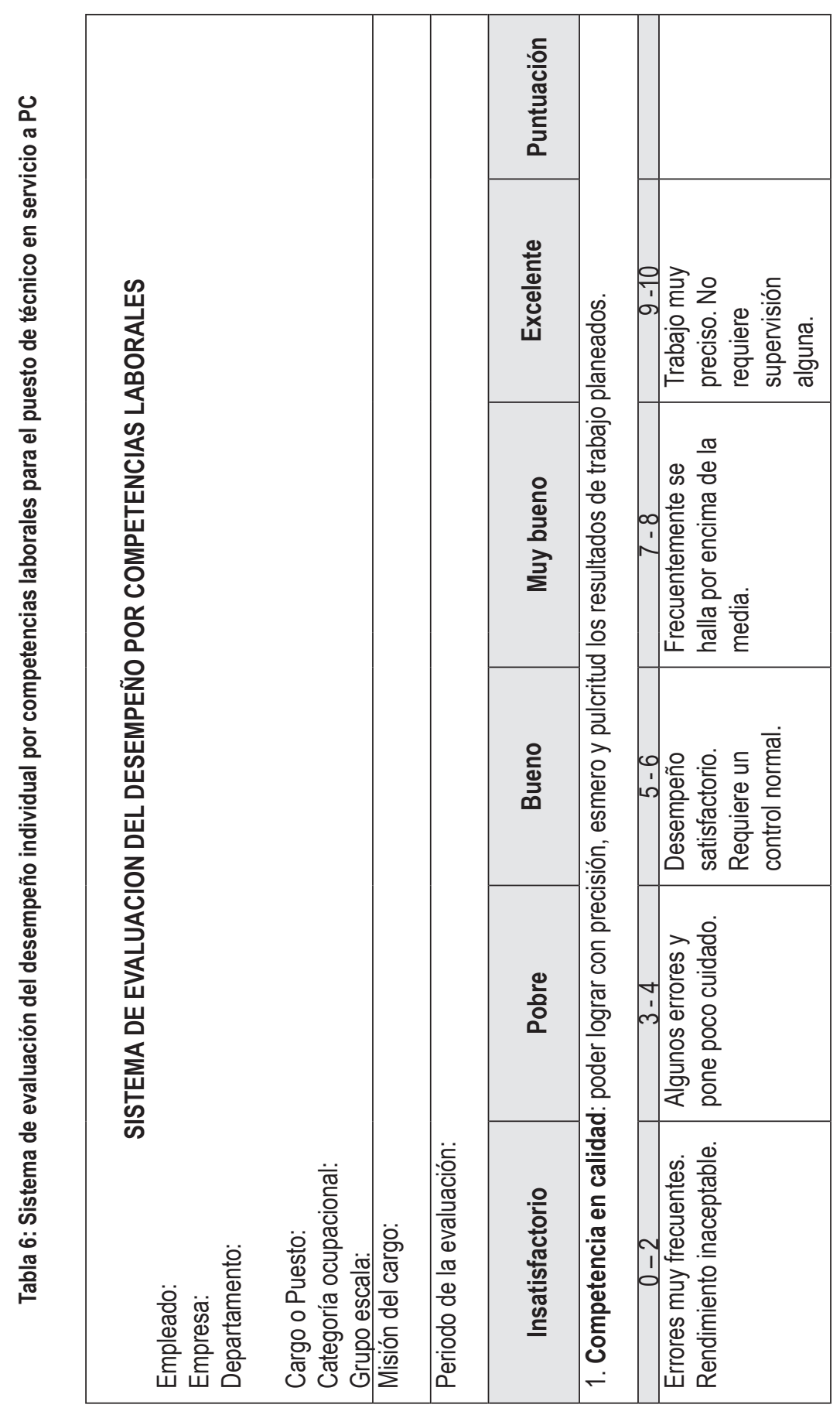


EVALUANDO DESEMPEÑOS: ALINEAMIENTO ESTRATÉGICO Y PRODUCTIVIDAD

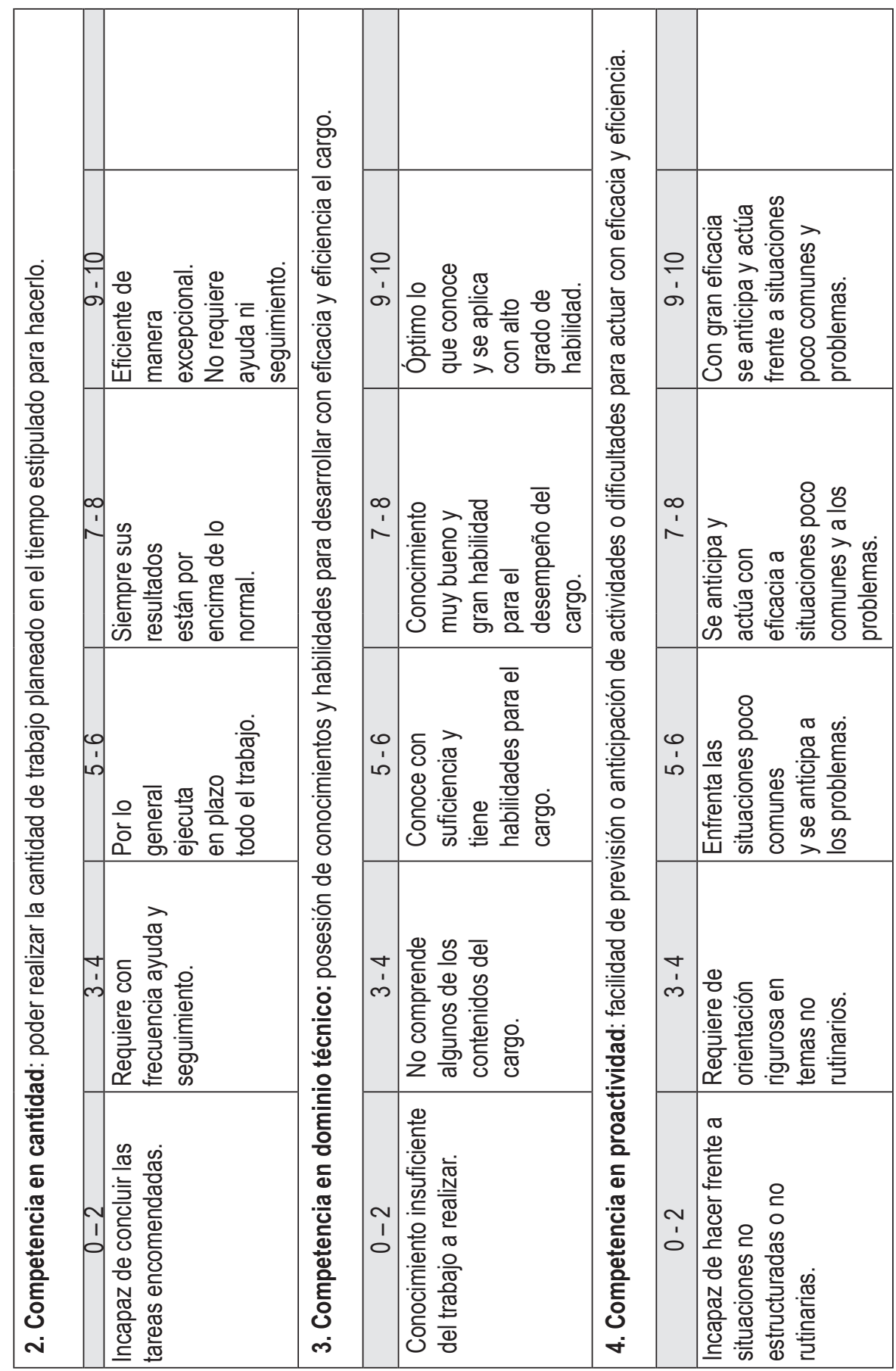

\begin{tabular}{l|l} 
ISSN 1541-8561 | 21 & 21
\end{tabular} 


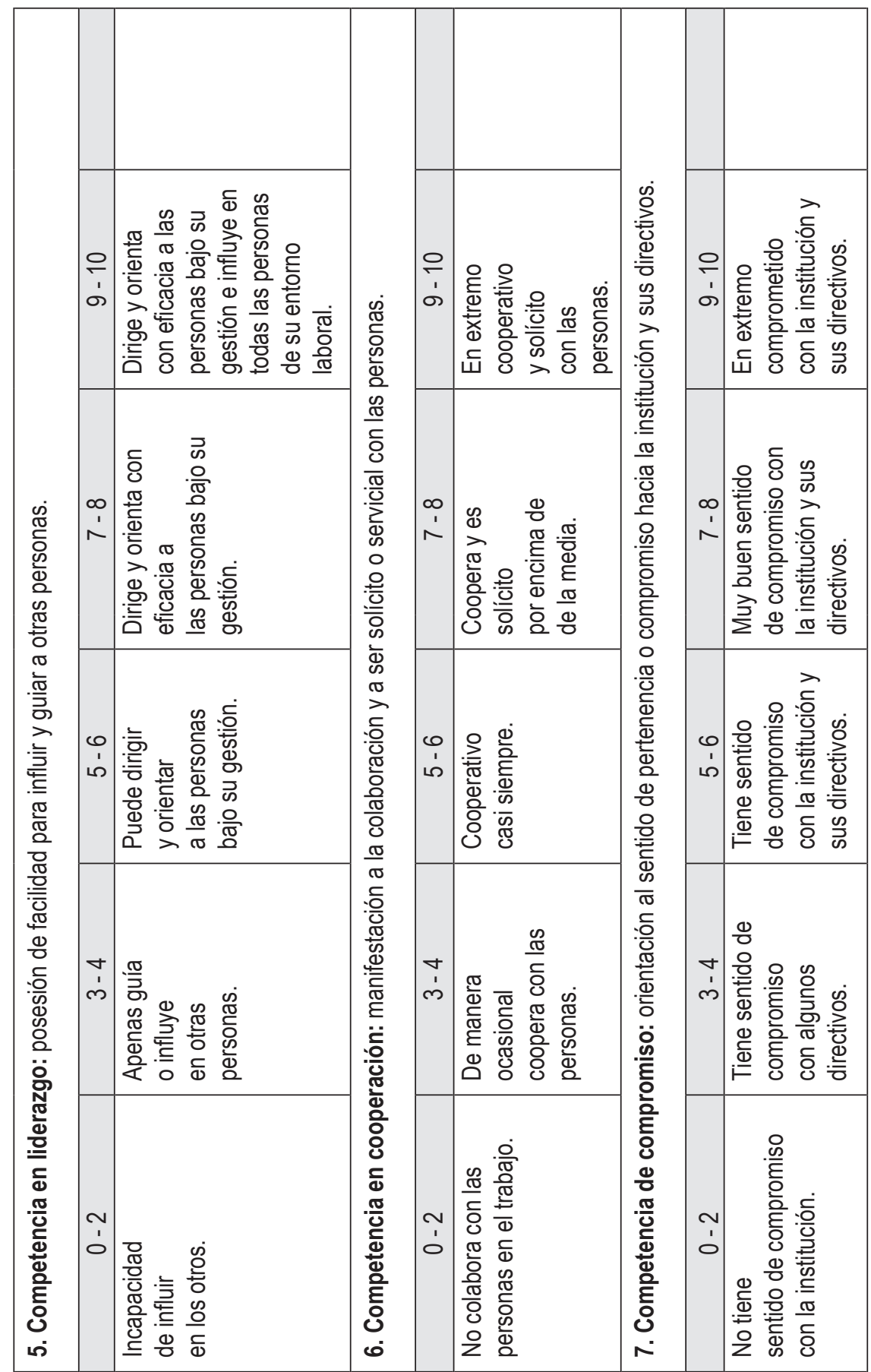


EVALUANDO DESEMPEÑOS: ALINEAMIENTO ESTRATÉGICO Y PRODUCTIVIDAD

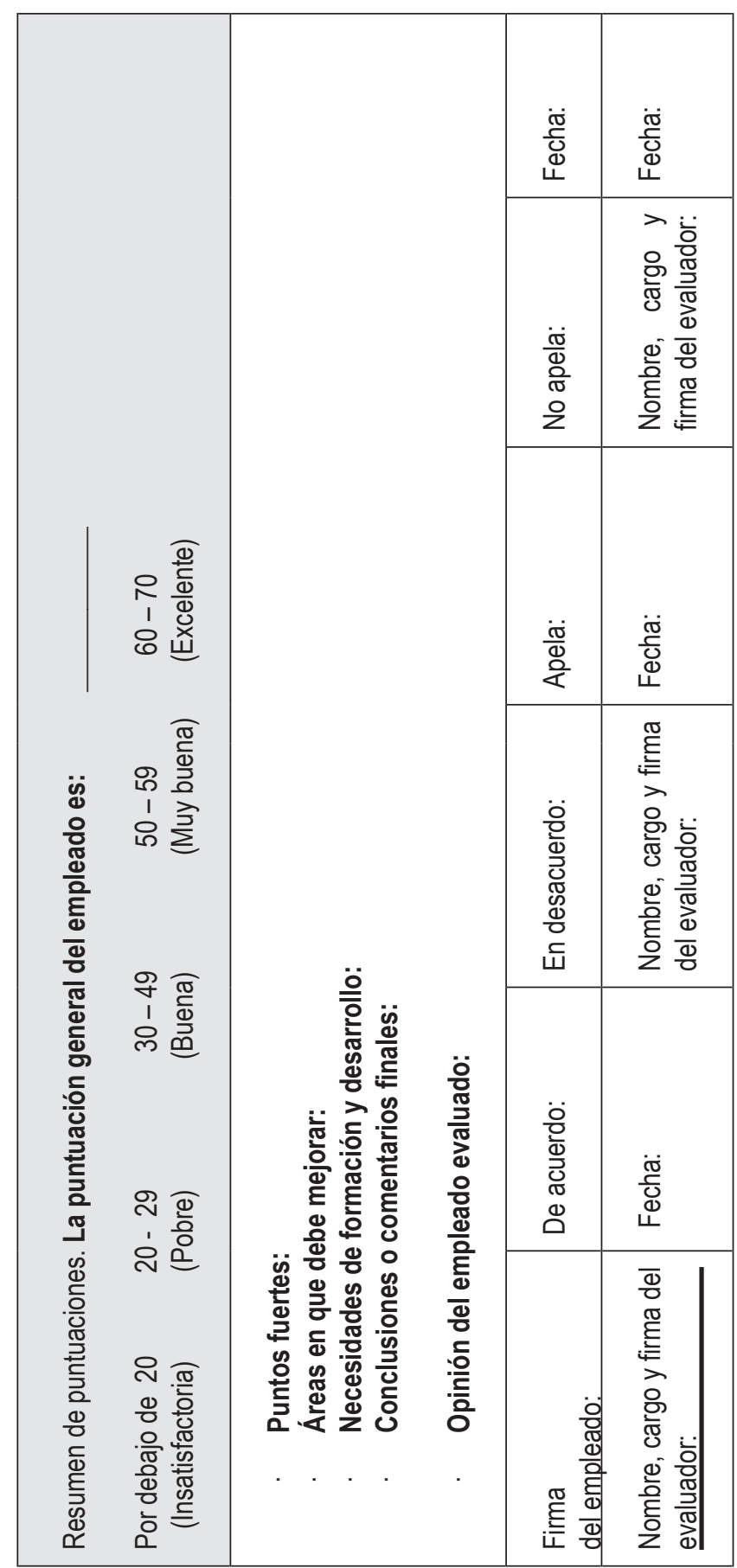




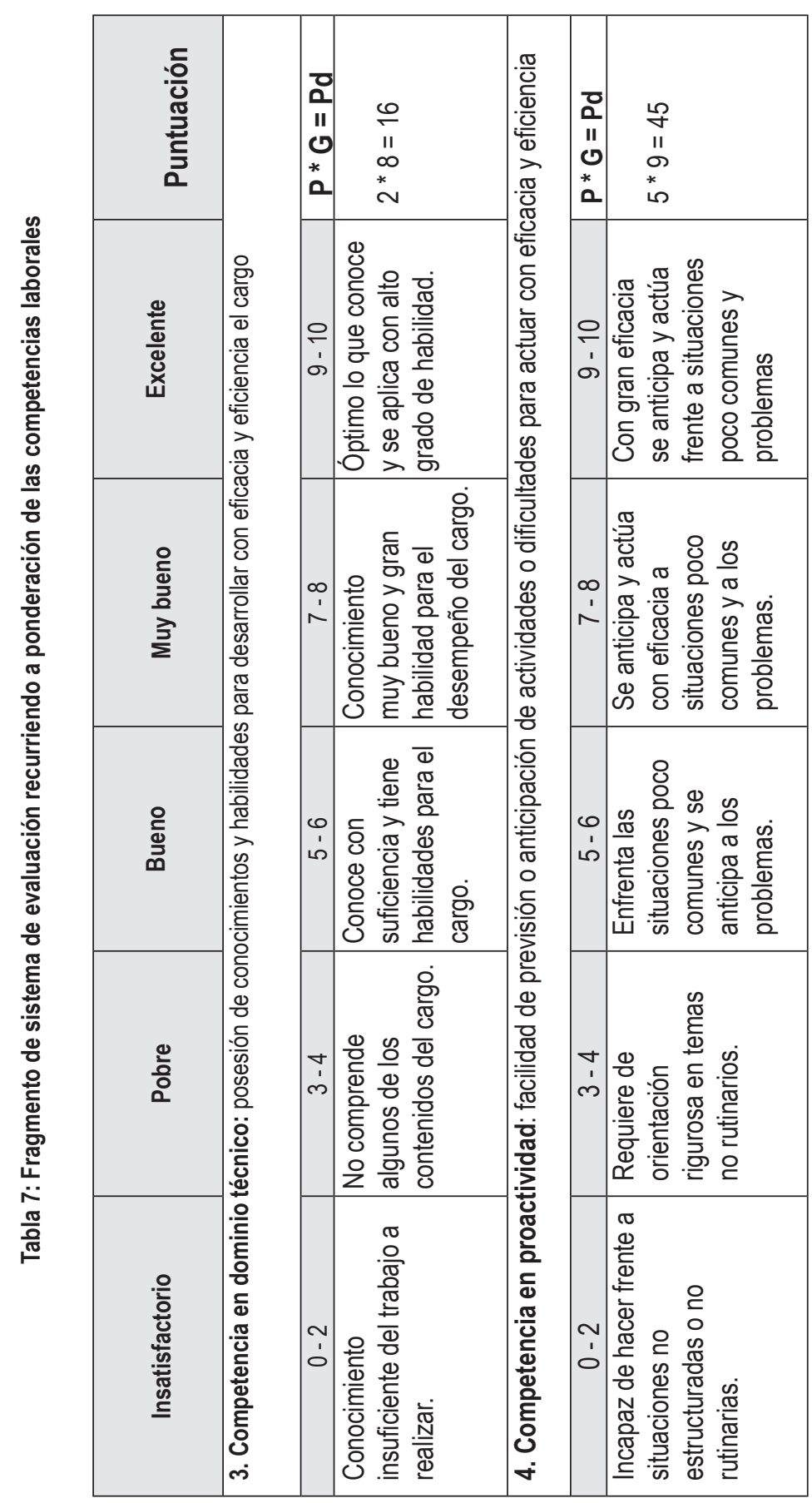


Ocurre en muchas ocasiones que las competencias varían en importancia o impacto respecto a la misión (no son lineales), es decir, poseen distintas ponderaciones, que la vida práctica impone (obsérvese ese comportamiento en el fragmento que revela la Tabla 7). Ello se supera si se considerara un factor de ponderación $(\mathrm{P})$ que multiplicado por el grado $(\mathrm{G})$ posibilite una puntuación diferenciada (Pd) para cada competencia, y en correspondencia una puntuación diferenciada total (Pdt) que resultaría de la sumatoria (å) de esas puntuaciones, teniéndose entonces:

$$
\begin{aligned}
& \mathbf{P d}=\mathbf{P} * \mathbf{G} \\
& \mathbf{P d t}=\mathbf{a} \mathbf{P d}
\end{aligned}
$$

Y por supuesto, se modificaría la escala clasificatoria de las puntuaciones que se reflejó en la Tabla 6, rompiendo así la estructura escalar 0 - 70 utilizada. La denominada puntuación diferenciada total o Pdt, bien podría asumir una escala 0 - 150, 0 - 200 o 0 - 280, para aludir a cualquier monto, dependiendo de las ponderaciones por las cuales convencionalmente opte el Comité de competencias (y no olvidar que el Delphi por rondas que se aplicó posibilitaba ponderaciones, solicitadas en su tercera ronda). Será la práctica la que indique los rangos cuantitativos a los cuales corresponderán los rangos cualitativos de Insatisfactorio, Pobre, Bueno, etc., y que definitivamente configurarán los expertos de la empresa.

Debe considerarse bien la necesidad de utilizar escalas cuantitativas, sin que esto signifique simplificar o reducir la necesaria complejidad que implica la evaluación del desempeño, tanto en su vertiente individual como organizacional, que habrá de considerarse como un todo integral u holístico, o como unidad dialéctica.

La necesidad de usar escalas cuantitativas (ordinales) no relega para nada aquí a los análisis cualitativos; contrariamente, contribuye a precisarlos más y justamente en su contexto empresarial u organizacional, a lo cual obliga el CMI utilizado, precisando que en el diseño del sistema de evaluación individual por competencias laborales, se tenga presente su correspondencia con objetivos, indicadores y metas, como se reflejó antes en la Tabla 5. 
No hay que forzar la introducción de escalas cuantitativas, reflejos de lo cualitativo; pero se impondrán ante el avance de la medición de los intangibles. Como bien plantearon los creadores del CMI: lo que no se mide, no se gestiona: “...si no puedes medirlo, no puedes gestionarlo" (Kaplan \& Norton, 1997). Pero esa medición no tiene que ver con la medición en la física clásica o el positivismo, que ha sido la predominante. Es relevante considerar en perspectiva holística la dialéctica de la evaluación del desempeño individual en su nexo con la evaluación estratégica organizacional, y así lo hace el procedimiento metodológico que se ha presentado.

\section{Conclusiones}

La práctica empresarial mundial de esta contemporaneidad ha verificado la ruptura o inexistencia de vínculos entre la GRH, la estrategia e indicadores de gestión organizacional. Esa ruptura no ha posibilitado que el desempeño individual tribute adecuadamente al desempeño estratégico organizacional, limitando o frenando a la productividad del trabajo. Es problema actual que la evaluación del desempeño individual sea opuesta (versus) a la evaluación estratégica, en tanto no están alineadas, integradas, o en correspondencia. Investigaciones realizadas en los últimos años posibilitaron apreciar la superación de esa ruptura mediante el diseño de un procedimiento metodológico, implicando que la evaluación del desempeño individual (conteniendo conductas estratégicas), se manifieste alineada a la evaluación organizacional estratégica, posibilitando aumento de productividad.

El procedimiento metodológico presentado partió de la asunción de una definición de competencia laboral, asumida en las NC 3000-3002: 2007. El proceso se inicia con la planificación estratégica empresarial, una vez decidida la dirección estratégica: habiéndose definido al menos la misión y los objetivos estratégicos. Con posterioridad se hacen los rediseños necesarios de los procesos de trabajo, para reajustar correspondencia de los mismos con la misión y objetivos estratégicos. Y atendiendo a los distintos procesos de trabajo reajustados o rediseñados se lleva a efectos el "Análisis y diseño de puestos", con la salida que es el descriptor o calificador de cargo, 
al cual se le adjuntará el perfil de competencias determinado, sintonizado con los objetivos estratégicos a alcanzar. Y a la vez que se concibe esa planeación, se diseña el control de gestión estratégica, considerando indicadores intangibles especialmente asociados a la productividad.

En el procedimiento metodológico ilustrado tiene relevancia el método utilizado en la determinación de las competencias laborales, por cuanto se exige de un conjunto de expertos que haya vivenciado el cargo en cuestión y posean dominio de la actual misión y objetivos estratégicos de la organización laboral. Ese método es el Delphi por rondas ponderado, aplicado al Comité de competencias que habrá de conformarse según el aludido procedimiento.

La recurrencia a escalas cuantitativas no ha implicado en esta experiencia desestimar análisis cualitativos, ni simplificar la complejidad comprendida por la evaluación del desempeño como unidad o conjunto, que es como se manifiesta en verdad en la práctica empresarial contemporánea. Esas escalas posibilitan la precisión del análisis cualitativo en contexto, al establecer correspondencia con objetivos, indicadores y metas que exige el CMI para controlar y evaluar el desempeño estratégico organizacional, ubicando a la evaluación del desempeño individual justamente en el contexto empresarial u organizacional.

El procedimiento metodológico que se ha presentado deberá ser aprehendido desde la perspectiva holística que refleja su entramado o complejidad, en su dialéctica de evaluación individual en su nexo con la evaluación estratégica organizacional. Manifestado ese nexo al implicar el alineamiento, integración o correspondencia, mediante las "conductas estratégicas" que llegan a establecerse en las competencias laborales en aras de la solución del problema planteado. A través del procedimiento presentado, la evaluación del desempeño individual y la evaluación del desempeño organizacional en su devenir se expresan como unidad. 


\section{Referencias}

BCG y WFPMA. (2010). Creating people advantage 2010 (How companies can adapt their HR practices for volatile times). Boston: The Boston Consulting Group, Inc. (BCG) y la World Federation of Personnel Management Associations (WFPMA).

BCG y WFPMA. (2008). Creating people advantage. Cómo afrontar los desafios de RRHH en todo el mundo hasta 2015 (Resumen Ejecutivo). Boston: The Boston Consulting Group, Inc. (BCG) y la World Federation of Personnel Management Associations (WFPMA).

Boxall, P., Purcell, J. y Wright, P. (2007). The handbook of human resource management. Oxford: Oxford University Press.

Boyatzis, R. (1982). The competent manager. New York: John Wiley \& Sons.

Chiavenato, I. (2002). Gestión del talento humano. Bogotá: McGrawHill.

Cuesta, A. (2010a). Nexos de las competencias laborales en la empresa: determinación, alineamiento estratégico y control de su gestión. Memorias. Trabajo presentado en la 15 Convención Científica de Ingeniería y Arquitectura, celebrada en el Palacio de las Convenciones de La Habana, del 29 de noviembre al 3 de diciembre de 2010. Editorial CUJAE: La Habana.

Cuesta, A. (2010b). Gestión del talento humano y del conocimiento. Bogotá: ECOE Ediciones.

Cuesta, A. (2001). O uso do metodo Delphi na criação de um modelo de competências. Revista Administração (RAUSP), 36(2), 2532.

Cuesta, A. y Valencia, M. (2010). La productividad del trabajo del trabajador del conocimiento. Revista de Gestión de la Universidad de Sao Paulo, 17(4), 421-436.

De Miguel, M. (2007). Tecnología para la planeación integral de los recursos humanos. Aplicación en entidades hoteleras del destino Holguín (Tesis de Doctorado en opción al grado científico de Doctora en Ciencias Técnicas). La Habana, CUJAE.

Dolan, S., Valle, R., Jackson, S. y Shuller, R. (2003). La gestión de los recursos humanos (preparando profesionales para el siglo XXI). Madrid: McGraw-Hill. 
Hernández, V. (2009). Evaluación y mejora de la actuación del personal y su incidencia en la calidad del servicio asistencial hospitalario (Tesis de Doctorado en opción al grado científico de Doctora en Ciencias Técnicas). La Habana, CUJAE.

Kaplan, R. y Norton, D. (2004). Strategy maps: Converting intangible assets into tangible outcomes. Boston: Harvard Business School Press.

Kaplan, R. y Norton, D. (2001). The strategy focused organization. Boston: Harvard Business School Press.

Kaplan, R. y Norton, D. (1997). The balanced scorecard. Boston: Harvard Business School Press.

Morales, A. (2006). Contribución para un modelo cubano de gestión integrada de recursos humanos (Tesis de Doctorado en opción al grado científico de Doctor en Ciencias Técnicas). La Habana: CUJAE.

NC 3000. 2007. Norma Cubana NC 3000: 2007: Sistema de gestión integrada de capital humano - Vocabulario. La Habana: Oficina Nacional de Normalización (NC). Recuperado de www.nc.cubaindustria.cu

NC 3001. 2007. Norma Cubana NC 3001: 2007: "Sistema de gestión integrada de capital humano - Requisitos. La Habana: Oficina Nacional de Normalización (NC). Recuperado de www.nc.cubaindustria.cu

NC 3002. 2007. Norma Cubana NC 3002: 2007: "Sistema de gestión integrada de capital humano - Implementación. La Habana: Oficina Nacional de Normalización (NC). Recuperado de www. nc.cubaindustria.cu

Nogueira, D., Medina, A. y Nogueira, C. (2009). Control de gestión y cuadro de mando integral: énfasis en la perspectiva financiera - aplicación en una empresa de servicios de informática. Revista Administração (RAUSP), 44(3), 222-235.

Programa de las Naciones Unidas para el Desarrollo (PNUD). (2010). Informe sobre Desarrollo Humano 2010 (Edición del Vigésimo Aniversario). Madrid: Mundi-Prensa.

Ramos, J. (2009). Perfeccionamiento del sistema de gestión de la capacitación de los cuadros y trabajadores de la organización CIMEX de la República de Cuba (Tesis de Doctorado en opción al grado científico de Doctor en Ciencias Técnicas). La Habana: CUJAE. 
Soltura, A. (2009). Tecnología de integración estratégica del sistema de competencias de la organización (Tesis de Doctorado en opción al grado científico de Doctor en Ciencias Técnicas). La Habana: CUJAE.

Valencia, M. (2010). Modelo de generación y transferencia de conocimiento para los procesos de dirección y gestión humana en PYMES del sector cárnico de la ciudad de Cali (Tesis de Doctorado en opción al grado científico de Doctor en Ciencias Técnicas). La Habana: CUJAE.

Werther, W. y Davis, K. (2001). Administración de personal y recursos humanos (5ta. ed.). México: Mc Graw Hill. 